\title{
First Report of Erythema Multiforme Minor Caused by Raloxifene Hydrochloride
}

\author{
Yurie Norimatsu ${ }^{a} \quad$ Yuta Norimatsu $^{\mathrm{a}, \mathrm{b}}$ \\ aDepartment of Dermatology, Ageo Daiichi Clinic for Internal Medicine and Pediatrics, \\ Ageo, Japan; bepartment of Dermatology, University of Tokyo Graduate School of \\ Medicine, Tokyo, Japan
}

\section{Keywords}

Raloxifene $\cdot$ Erythema multiforme minor $\cdot$ Osteoporosis $\cdot$ Skin biopsy

\begin{abstract}
Raloxifene is a drug used in postmenopausal women with osteoporosis. Although hot flashes are known side effects of raloxifene, to the best of our knowledge, erythema multiforme (EM) minor has not been previously reported. Herein, we report about a 74-year-old woman who developed EM minor after the drug alfacalcidol was changed to raloxifene to treat osteoporosis. Skin biopsy revealed a suspicious eczematous drug reaction. The drug-induced lymphocyte stimulation test showed a positive result. The stimulation index was 2.2, and there were no other suspected drugs. Based on these results, we diagnosed the condition as EM minor caused by raloxifene. The patient's symptoms disappeared after the use of antihistamine drugs and topical steroids. In conclusion, raloxifene can cause EM minor in rare cases.
\end{abstract}

\section{Introduction}

Raloxifene is a benzothiophene selective estrogen receptor modulator (SERM). SERMs are designed to have both estrogen agonist and antagonist effects [1,2]. Estrogen is known to cause dermatitis $[3,4]$. However, estrogen dermatitis can be treated using tamoxifen, a type of SERM [4]. Therefore, it was considered that the pathophysiology of estrogen dermatitis was not involved in this case. Similarly, tamoxifen is known to cause toxic epidermal necrolysis [5]. Thus, SERMs can cause drug eruptions. 


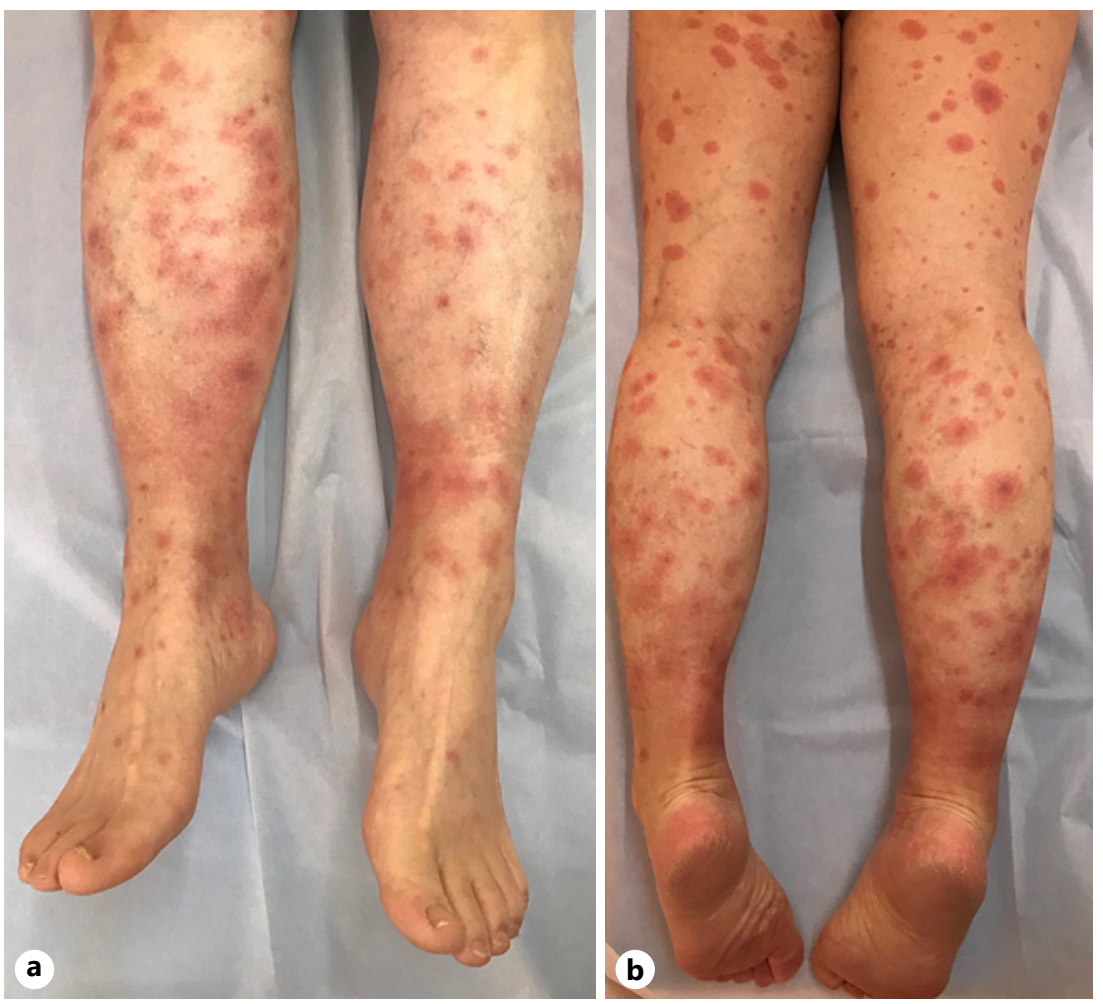

Fig. 1. a Front of the lower limbs at the first visit. $\mathbf{b}$ Back of the lower limbs at the first visit.

To the best of our knowledge, erythema multiforme (EM) minor induced by raloxifene has not been previously reported. In this report, we present the first case of EM minor induced by raloxifene in Japan.

\section{Case Presentation}

A 74-year-old woman with a history of hypertension, hyperlipidemia, and osteoporosis had been taking benidipine, pitavastatin, and alfacalcidol for more than a few years; however, alfacalcidol was changed to raloxifene owing to inadequate treatment response for osteoporosis (15 days before presentation). A rash began to appear mainly on the lower limbs 3 days before presentation. She visited our internal medicine clinic (Ageo Daiichi Clinic for Internal Medicine and Pediatrics) 2 days before presentation and was instructed to stop taking raloxifene and start taking topical betamethasone butyrate propionate. She then visited our dermatology clinic because of poor improvement in her condition (day 0). Erythema, which was observed as a target lesion, was scattered around the lower limbs, abdomen, back, and face (shown in Fig. 1). Since she experienced no enanthem, prior infection, or fever, the condition was diagnosed to be EM minor [6].

Skin biopsy was performed on day 0 , which revealed vacuolar degeneration in the base of the epidermis and mild lymphocyte and eosinophil infiltration in the upper dermis (shown in Fig. 2). Since the patient's rash gradually expanded and became palpable, betamethasone butyrate propionate was changed to a very potent steroid, clobetasol propionate (external use), from day 0 , and oral antihistamines were commenced. A blood test was performed on day 2 , which revealed normal results. The drug-induced lymphocyte stimulation test

\section{Karger'}


Case Reports in Dermatology
Case Rep Dermatol 2021;13:445-449

DOI: $10.1159 / 000519029$

C 2021 The Author(s). Published by S. Karger AG, Basel www.karger.com/cde

Norimatsu and Norimatsu: EM Minor Due to Raloxifene

Fig. 2. Hematoxylin-eosin staining $(\times 200)$.
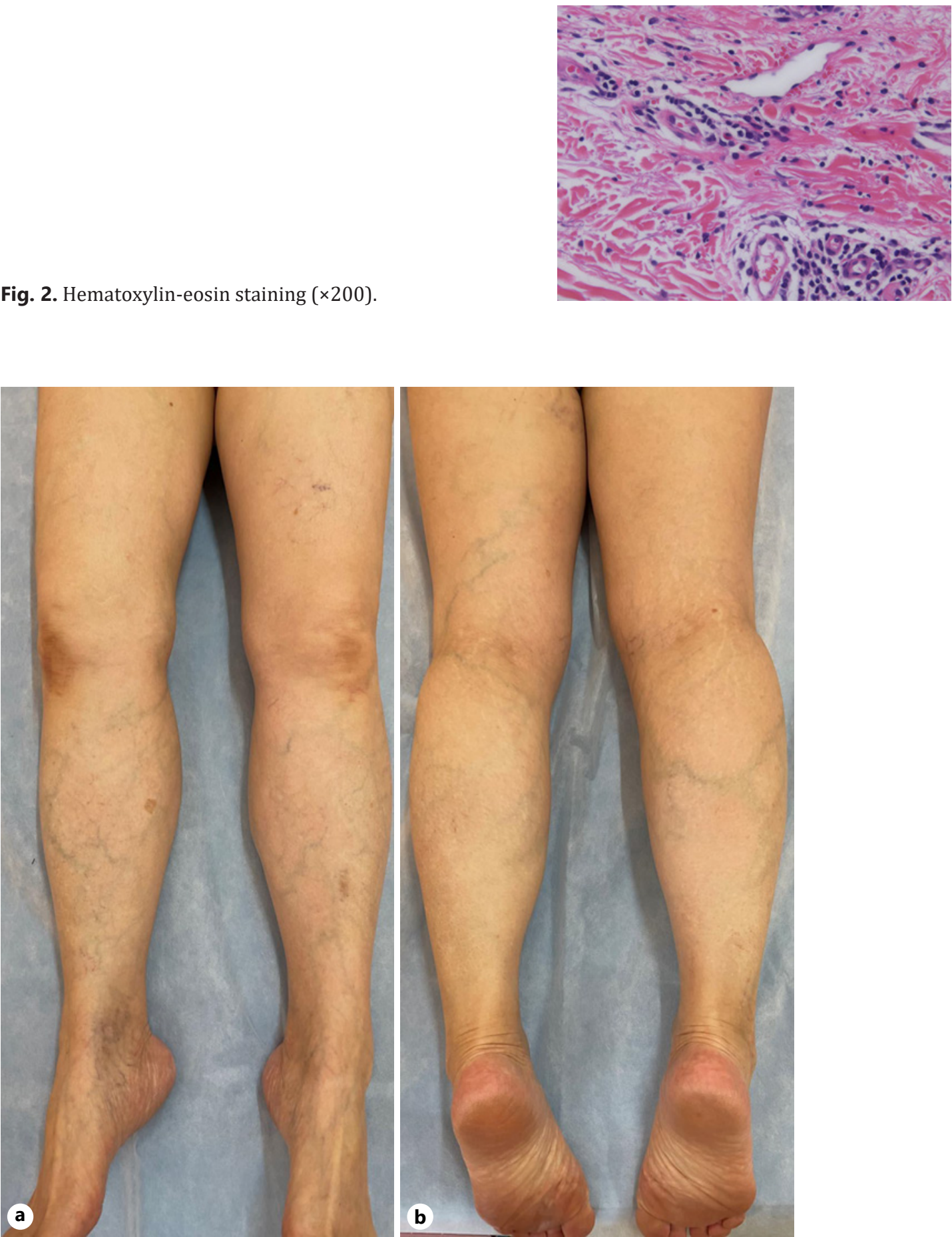

Fig. 3. a Front of the lower limbs at the 6-month follow-up. b Back of the lower limbs at the 6-month follow-up.

performed on day 7 showed positive results (control, 80; raloxifene, 174; stimulation index, 2.2). Erythema was mild by day 7, and no recurrence was observed after a 6-month follow-up (shown in Fig. 3).

\section{Karger' ${ }^{\prime \prime}$}




\section{Discussion}

We experienced a case of EM minor caused by raloxifene. EM has been found to be associated with previous infections in $30 \%$ of cases, with the administration of drugs in $30 \%$ of cases, and with a combination of the 2 factors in $30 \%$ of cases; no specific etiology was found in $10 \%$ of cases [7]. Our patient had no prior infection but had a history of drug changes. Therefore, we attributed her EM minor to the administration of drugs, specifically raloxifene.

Although a skin biopsy may show eosinophil infiltration, it is difficult to confirm this as a drug eruption only based on skin biopsy results [8]. However, in our case, there were no other suspected drugs, except raloxifene, and the drug-induced lymphocyte stimulation test result was consistent with that of raloxifene-induced drug eruption. Rapid progress with the exclusive administration of antihistamines and topical steroids after discontinuing raloxifene is considered to be consistent with the clinical presentation of drug eruption.

The World Health Organization-Uppsala Monitoring Centre system was used to evaluate the drug eruption [9]. In our case, the pharmacological mechanism was unknown; therefore, the causality category of this case is considered to correspond to the probable level.

In conclusion, raloxifene can cause drug eruptions in rare cases. Raloxifene-induced drug eruption presents as EM minor type, and the evaluation of drug history is important for establishing an accurate diagnosis.

\section{Statement of Ethics}

This study protocol was reviewed, and the need for approval was waived by the Ethics Committee of the University of Tokyo Hospital. Written informed consent was obtained from the patient for publication of the details of their medical case and any accompanying images.

\section{Conflict of Interest Statement}

The authors have no conflicts of interest to declare.

\section{Funding Sources}

The cost of submitting this paper will be covered by private funds.

\section{Author Contributions}

Both authors are the patient's doctors and wrote the manuscript.

\section{Data Availability Statement}

The data that support the findings of this study are not publicly available due to their containing information that could compromise the privacy of research participant but are available from Yuta Norimatsu upon reasonable request.

\section{Karger'}




\section{References}

1 Sato M, Glasebrook AL, Bryant HU. Raloxifene: a selective estrogen receptor modulator. J Bone Miner Metab. 1994 Dec;12(S2):S9-20.

2 Snyder KR, Sparano N, Malinowski JM. Raloxifene hydrochloride. Am J Health Syst Pharm. 2000 Sep;57(18): 1669-75.

3 Fukayama M, Asano Y, Shinozaki-Ushiku A, Sato S. Estrogen dermatitis: case report and examination of estrogen receptor- $\beta$ in the skin. J Dermatol. 2019 Mar;46(3):263-6.

4 Shelley WB, Shelley ED, Talanin NY, Santoso-Pham J. Estrogen dermatitis. J Am Acad Dermatol. 1995 Jan;32(1): 25-31.

5 Madabhavi I, Revannasiddaiah S, Patel A, Anand A. Toxic epidermal necrolysis with the use of tamoxifen. BMJ Case Rep. 2015 Apr 15;2015:bcr2014209102.

6 Stampien TM, Schwartz RA. Erythema multiforme. Am Fam Physician. 1992;46:1171-6.

7 Martín Mateos MA, Roldán Ros A, Muñoz-López F. Erythema multiforme: a review of twenty cases. Allergol Immunopathol. 1998 Nov-Dec;26(6):283-7.

8 Ramdial PK, Naidoo DK. Drug-induced cutaneous pathology. J Clin Pathol. 2009 Jun;62(6):493-504.

9 World Health Organization. The use of the WHO-UMC system for standardised case causality assessment [Internet]. Geneva: World Health Organization; 2013 [cited 2021 Aug 11]. Available from: https://www.who. int/publications/m/item/WHO-causality-assessment. 